\title{
A pendulum test as a tool to evaluate viscous friction parameters in the equine fetlock joint
}

\author{
Prisca Noble ${ }^{\mathrm{a}, *}$, Geoffroy Lumay ${ }^{\mathrm{b}}$, Marc Coninx ${ }^{\mathrm{c}}$, Bernard Collin ${ }^{\mathrm{a}}$, Adrien Magnée ${ }^{\mathrm{d}}$, \\ Jacqueline Lecomte-Beckers ${ }^{\mathrm{d}}$, Jean M. Denoix ${ }^{\mathrm{e}}$, Didier Serteyn ${ }^{\mathrm{c}}$ \\ a Department of Morphology and Pathology, Division of Anatomy, Liège University Faculty of Veterinary Medicine, Boulevard de Colonster, 20, B43, B-4000 Liège, Belgium \\ ${ }^{\mathrm{b}}$ GRASP, Group for Research and Applications in Statistical Physics, Liège University Faculty of Sciences, Boulevard de Colonster, 20, B43, B-4000 Liège, Belgium \\ ${ }^{\mathrm{c}}$ Department of Clinical Sciences, Equine Clinic, Liège University Faculty of Veterinary Medicine, Boulevard de Colonster, 20, B43, B-4000 Liège, Belgium \\ ${ }^{\mathrm{d}}$ Department of Aerospace Industry and Mechanics, Division of Metallurgy and Materials Science, Liège University Faculty of Applied Science, Chemin des chevreuils, 1, B52, \\ B-4000 Liège, Belgium \\ e CIRALE, Centre d'imagerie et de recherche en affections locomotrices équines, RD675 14430 Goustranville, France
}

\section{A R T I C L E I N F O}

\section{Article history:}

Accepted 17 March 2010

\section{Keywords:}

Equine articular cartilage

Viscous damping coefficient

Friction coefficient

Lubrication

Pendulum test

\begin{abstract}
A B S T R A C T
An equine fetlock joint pendulum test was studied and the influence of post mortem time and intra-articular lipid solvent on the viscous frictional response examined. Fresh equine digits (group 1, $n=6$ controls; group 2, $n=6$ lipid solvent) were mounted on a pendulum tribometer. Assuming that pendular joint damping could be modelled by a harmonic oscillator fluid damping (HOFD), damping time $(\tau)$, viscous damping coefficient $(c)$ and friction coefficient $(\mu)$ were monitored for $5 \mathrm{~h}$ under experimental conditions $\left(400 \mathrm{~N} ; 20^{\circ} \mathrm{C}\right)$. In all experiments, pendular joint damping was found to follow an exponential decay function $\left(R^{2}=0.99714\right)$, which confirmed that joint damping was fluid.

The evolution of $\tau, c$ and $\mu$ was found to be significantly $(P<0.05)$ different in the two groups, with a decrease in $\tau$ and an increase in $c$ and $\mu$ that was faster and more prominent in digits from group 2. It was concluded that pendular joint damping could be modelled by a HOFD model. The influence of post mortem time on results suggested that, ideally, joint mechanical properties should only be tested on fresh cadavers at the same post mortem time. Moreover, the addition of lipid solvent was found to be responsible for upper viscous friction parameters and for a reduced damping time, which suggested that articular lubricating ability was compromised. This equine pendulum test could be used to test the efficacy of various bio-lubricant treatments.
\end{abstract}

(C) 2010 Elsevier Ltd. All rights reserved.

\section{Introduction}

The synovial joint is a complex tribological structure, which supports and redistributes joint contact forces and facilitates low friction and low wear. The connective tissue of the synovial joint, a triplex of cartilage-synovial fluid-cartilage, is essential to function and provides a sophisticated lubricated system.

In recent years, the cartilage lubrication mechanism has been shown to depend significantly on the pressure of the cartilage interstitial water, which supports most of the joint contact load and helps to shift it away from the collagen-proteoglycan matrix, thereby producing a low frictional response (Ateshian, 1997, 1998; Krishnan et al., 2004; Caligaris and Ateshian, 2008). Frictional forces would be significant only over the portion of the load transferred across the solid matrices of the opposing articular layers; thus, as long as the interstitial fluid pressure remains elevated, supporting most of the load, the friction should remain low (Atesh-

\footnotetext{
* Corresponding author. Tel.: +32 43664061.

E-mail address: pnoble@ulg.ac.be (P. Noble).
}

ian, 2009). This mechanism, called biphasic boundary lubrication or self-pressurised hydrostatic lubrication, is related to cartilage composition.

Articular cartilage consists of water (68-85\% weight), fibrillar matrix of type II collagen (10-20\%), proteoglycans (5-10\%) and chondrocytes. Due to the negative polarity of proteoglycans, which attracts more cations than anions into the tissue to maintain electroneutrality, a net electrolytic imbalance is created between the cartilage interstitial fluid and the surrounding tissue bath. This phenomenon produces a difference in osmotic pressure whereby the interstitial fluid exhibits a higher pressure than the external solution (Ateshian, 2009). A complementary approach, called boundary lubrication, has however been proposed, which describes the lubricating ability of the components of synovial fluid (SF). The boundary lubricants are adherent to apposed surfaces and thereby prevent adhesion and wear.

Three types of boundary lubricant are considered to be involved, individually or in combination, in synovial joints, namely, hyaluronan (HA), lubricin and surface-active phospholipids (SAPLs) (Jay, 1992; Schwarz and Hills, 1998; Elsaid et al., 2005; Bell et al., 
2006). Hyaluronan is an un-branched high-molecular-weight (500$3800 \mathrm{kDA}, 1-4 \mathrm{mg} / \mathrm{mL}$ ) anionic disaccharide consisting of alternating units of glucuronic acid $\beta(1-3)$ and $\mathrm{N}$-acetylglucosamine $\beta$ (14) (Forsey et al., 2006). HA is very hydrophilic $\left(50,000 \mathrm{H}_{2} \mathrm{O}\right.$ molecules for $1 \mathrm{HA}$ ), which gives SF a high viscosity (Trunfio Sfarghiu, 2006) and it has been recognised as the key molecule responsible for the rheological properties of SF (Tadmor et al., 2002).

Lubricin is a mucinous glycoprotein $(240 \mathrm{kDA}, 0.02 \mathrm{mg} / \mathrm{mL})$ that consists of four components: (1) a vitronectin-like domain which confers a molecular adhesive role; (2) a mucin-like domain in which the abundance of negatively charged sugars creates strong repulsive hydration forces that enable the protein to act as a boundary lubricant; (3) a haemopexin-like domain that confers a lipidic antioxidant role, and (4) a linkage protein (Schvartz et al., 1999; Tolosano and Altruda, 2002; Rhee et al., 2005). Lubricin is now recognised as playing a major protective role in preventing cartilage wear and synovial cell adhesion and proliferation (Rhee et al., 2005).

SAPLs are amphiphilic molecules $(0.75 \mathrm{kDA}, 3 \mathrm{mg} / \mathrm{mL})$ that are composed of a hydrophobic domain consisting of two fatty acid chains and a hydrophilic domain (Sarma et al., 2001). According to their hydrophobic domain, SAPLs are classified into two types: (1) unsaturated species (57\%) and (2) saturated species (43\%) (Sarma et al., 2001). Lipids are not miscible in SF water and SAPLs are therefore organised into 3-7 phospholipid bilayers on the surface of cartilage in joints, causing SF gel to be imprisoned between them (Schwarz and Hills, 1998). By attaching SAPLs on the SF gel and on the cartilage, and by performing its lipidic antioxidant role, lubricin would provide SAPLs stability (Trunfio Sfarghiu, 2006).

The impact of SAPL organisation and composition on boundary lubrication is important since the frictional response will decrease significantly when the number of phospholipid bilayers increases (Higaki et al., 1997), while the degree of un-saturation within fatty acid chain residues will reduce friction even further (Foy et al., 1999).

The role of SAPLs is debatable due to their instability. Indeed, unsaturated SAPLs are present as bilayers in an antioxidant and hydrophilic environment, such as the joint (Trunfio Sfarghiu, 2006), where the tension of oxygen $\left(\mathrm{O}_{2}\right)$ is low (7\%) (Grimshaw and Mason, 2000; Schneider et al., 2007). When SAPLs are present in ambient air $\left(\mathrm{O}_{2} 21 \%\right)$, the phospholipid bilayers are transformed into hydrophobic phospholipid mono-layers and the unsaturated connections are oxidised in oxirane (oxygen fixation) and then in diol $\left(\mathrm{H}_{2} \mathrm{O}\right.$ fixation) (Trunfio Sfarghiu, 2006). Singly or in combination, these phenomena act to decrease the role of SAPLs in boundary lubrication.

It is therefore apparent that to investigate the properties of articular cartilage we require a realistic joint model. Some studies have maintained conditions found within the joint to test the boundary lubricating ability of novel intra-articular treatments (Elsaid et al., 2005; Teeple et al., 2007, 2008), although not SAPLs. There is even less information known about the properties of articular cartilage when the joint atmosphere is conserved and a joint viscous friction model used.

The present study was the first attempt to measure viscous friction parameters on a whole equine metacarpophalangeal joint (MPJ), conserving the structural integrity of the joint and testing the influence of post mortem time and intra-articular lipid solvent.

\section{Materials and methods}

Specimen preparation

Twelve warmblood horse carcasses from a local abattoir were used in this study. The bodyweight (BW) ranged from 350 to $450 \mathrm{~kg}$ (mean $400 \mathrm{~kg}$ ), and we collected the right distal limbs (cut at the middle carpus) immediately after slaughter. These were classified as group $1(n=6)$ or group $2(n=6)$. For each specimen, the skin and the musculature were left intact, the hoof was trimmed in order to have a linear pastern-coffin axis and, using a power saw, the metacarpus was cut transversally $2 \mathrm{~cm}$ distal to the carpometacarpal joint.

The specimen was then fixed on the pendulum apparatus, completely immobilising the interphalangeal joints and leaving free the MPJ (Fig. 1). The hoof was screwed using six screws and the interphalangeal joints were clamped onto the static portion of the apparatus. The pendulum was fixed and adjusted on the metacarpus using eight compression screws. Biomechanical tests commenced within 90 min of slaughter.

Description of the pendulum test

A lateral-view of the pendulum apparatus is shown in Fig. 1. The apparatus was used to measure the pendular damping time $(\tau)$ or the time duration from onset angle to resting angle, the viscous damping coefficient $(c)$ and the friction coefficient $(\mu)$.

The pendulum was designed to swing the metacarpus relative to the fixed pastern-hoof segment while a compressive load of 400 Newtons (N) was applied across the MPJ. The motions of the MPJ were recorded using a camera coupled to light emitting diodes (LEDs). The acquisition system was Image,${ }^{1}$ an open-source program that was developed by the National Institutes of Health. ${ }^{2}$ Four LEDs were rigidly mounted on the pendulum to document MPJ motion. The wire attachments to the LEDs were maintained free of tension. The locations of the LEDs were referenced to a terrestrial based coordinate system. Within this coordinate system, the metacarpus was identified as a rigid body and pendulum motion was described relative to the fixed terrestrial referential. The MPJ is a condylar joint, and pendulum motion was characterised as a flexion-extension motion.

\section{Description of mechanical measurements and calculations}

After immobilising the MPJ in $5^{\circ}$ extension using an attached cord between the horizontal load bar and the vertical beam, we cut the cord using a flame so that the metacarpus was released, fell and oscillated freely between flexion and extension until it stopped. The inherent viscoelastic properties of the joint and the surrounding tissues, coupled to the mass of the moving loaded metacarpus, caused the metacarpus to come to rest close to the equilibrium position (static).

As shown in Fig. 2, several variables could be derived from the kinematics of each trial of the pendulum test. We measured the following displacement and timing parameters: the angle at the start of the test (onset angle), the peak angle of the cycle $n\left(\theta_{n}\right)$, the time duration from onset angle to resting angle or pendular damping time $(\tau)$, and the period of the cycle $(T)$.

Assuming that pendular joint damping acted as a harmonic oscillator with fluid damping (HOFD), the MPJ kinematic data of each trial were used to compute the variations of viscous friction parameters for the duration of each experiment. The viscous damping coefficient $(c)$ and friction coefficient $(\mu)$ were calculated using the following equations as reported by Lin and Rymer (1991) and Crisco et al. (2007):

$\zeta=\frac{c}{2 \sqrt{J K^{\prime}}}=\sqrt{\frac{(\ln D)^{2}}{4 \pi^{2}+(\ln D)^{2}}}$

where $J$ is the sagittal moment of inertia applied to the metacarpus complex rotation around the MPJ axis;

$K^{\prime}=K+\frac{m g l}{2}$

and $m$ is the metacarpus complex mass, $g$ is the gravity acceleration and $l$ is the distance of the metacarpus complex centre of mass from the MPJ axis; $D=\frac{\theta_{n}}{\theta_{n}}$ is the ratio of the peak angle of one cycle $\left(\theta_{n}\right)$ to the peak angle of the following cycle $\left(\theta_{n+1}\right)$.

$\omega=\sqrt{\frac{K^{\prime}}{J}}=\frac{2 \pi}{T}$

where $T$ is the period of one cycle (see Fig. 2).

The estimations of $J$ and of mass characteristics ( $m$ and $l$ ) were obtained for each subject according to Huygens' theory:

$J_{\Delta}=J_{G \Delta}+m d^{2}$

where solid inertial moment around an axis $\Delta$ is equal to the inertial moment around an axis going through the solid inertial centre of mass $\left(J_{G A}\right)$, added to the solid mass multiplied by the second power of the distance between the two axes $\left(m d^{2}\right)$.

$\theta_{n}=\theta_{0}\left[e^{-2 n \zeta}-\delta \frac{1+e^{-\zeta}}{1-e^{-\zeta}}\left(1-e^{-2 n \zeta}\right)\right]$

$\delta=\frac{\mu m g r T^{2}}{4 \pi^{2} J \theta_{0}}$

\footnotetext{
1 http://rsbweb.nih.gov/ij/

2 http://www.nih.gov/.
} 


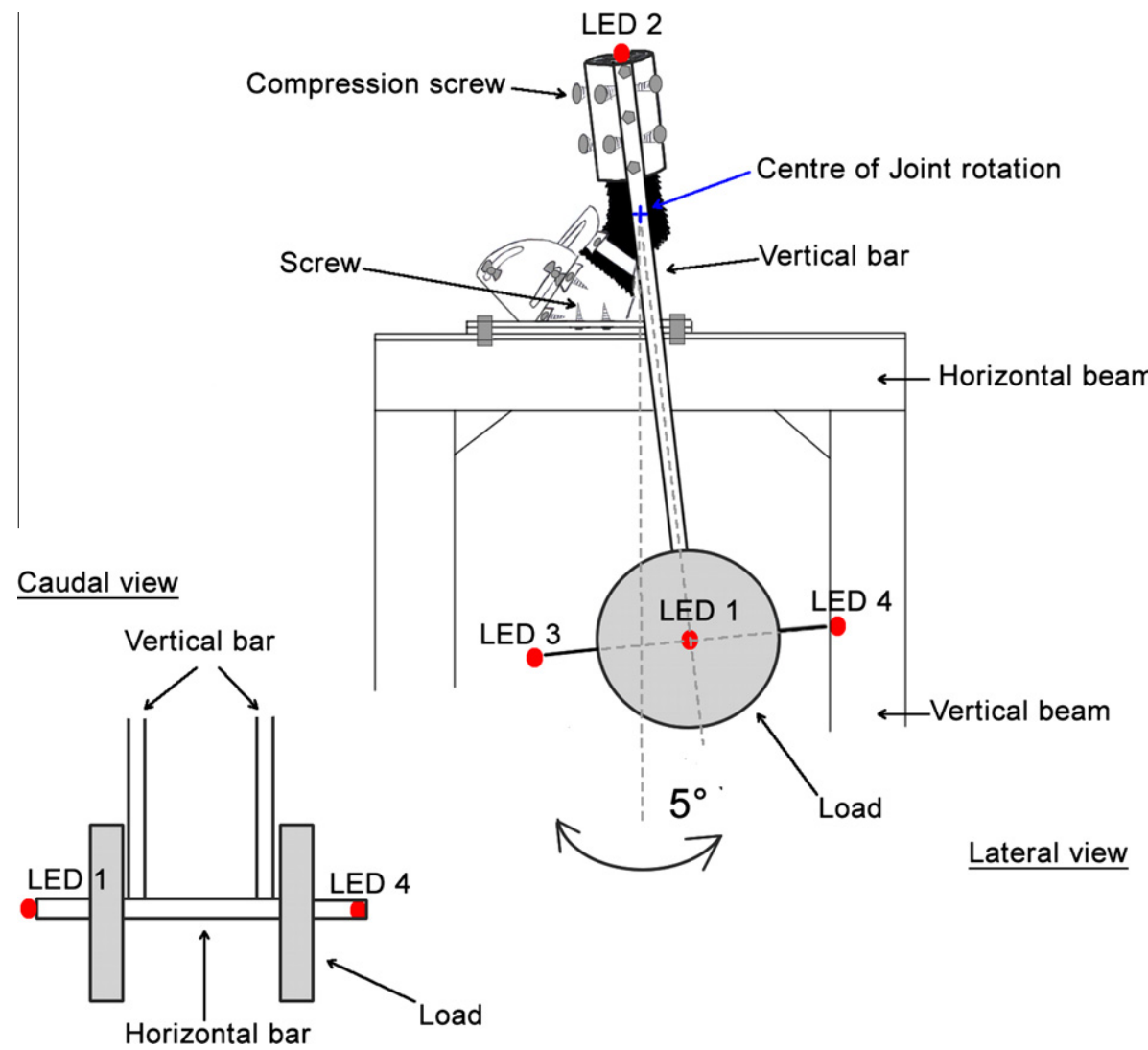

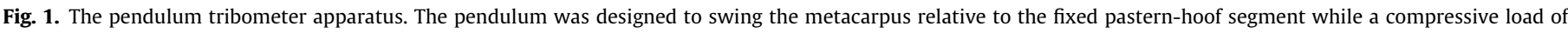

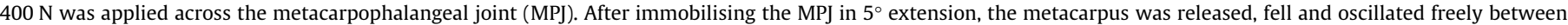

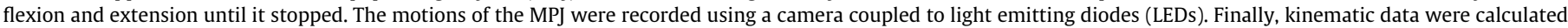
to estimate viscous frictional response.

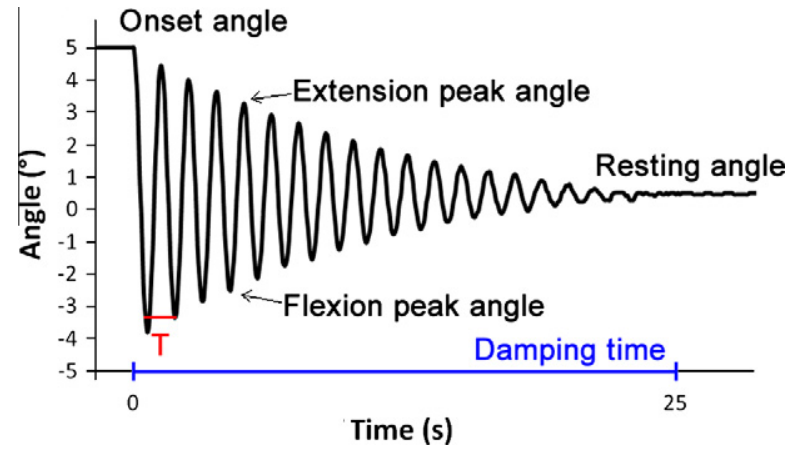

Fig. 2. Typical metacarpophalangeal joint flexion-extension response showing onset angle, resting angle, the period of the first cycle $(T)$ and damping time.

Using Eqs. (1) and (2), the values of the viscous damping coefficient were obtained as follows:

$c=2 \cdot \zeta \cdot \omega \cdot J$

Using Eqs. (3) and (4), the values of the friction coefficient were obtained as follows:

$\mu=\frac{\delta 4 \pi^{2} J \theta_{0}}{m g r T^{2}}$

\section{Tested parameters}

All experiments were performed at room temperature $\left(20^{\circ} \mathrm{C}\right)$. Two sets of experiments were defined in this study (Table 1). Experiment E1 investigated the evolution of the joint mechanical properties after slaughter and we measured $\tau, c$ and $\mu$ in group $1(n=6)$, for four trials (at 90, 190, 290 and 390 min post mortem). For each trial, $c$ and $\mu$ were averaged from the first 10 oscillation peaks. In order to disorganise SAPLs in experiment E2, we investigated the evolution of the joint mechanical properties before and after an injection of $2.5 \mathrm{~mL}$ Norvanol D (denatured ethanol 95\%, VWR international PROLABO) given in the palmar recess of each MPJ using a syringe with a 21 GA needle. We measured $\tau, c$ and $\mu$ in group $2(n=6)$, for five trials (at $90 \mathrm{~min}$ before and after alcohol addition, 190, 290 and $390 \mathrm{~min}$ post mortem). For each trial, $c$ and $\mu$ were averaged from the first 10 oscillation peaks.

Between every trial, each specimen remained fixed on the apparatus to avoid any adjustment error. The MPJ was relaxed using a hydraulic jack to support the loaded horizontal bar. All MPJs were cut open just after the experiments to reveal the articular surface (smooth or rough, stiff or compressible with a bluish or yellowish tint) and the synovial fluid. We then undertook a macroscopic examination.

Statistical analysis

The experimental values of pendular decay, corresponding to the peak values of the LED displacements in direction $x$, were fitted using two different models, one exponential and the other linear. An $F$-test was then applied to find out which model was the best fit for the same pendular decay (OriginPro 8). The statistical significance of the differences in $\tau, c$ and $\mu$ for experiment E2 before and after the addition of alcohol were determined using one-way analysis of variance (ANOVA) with repeated measurements to compare values (two levels) in group 2 . The statistical significance of the differences in $\tau, c$ and $\mu$ for experiments E1 and E2 was determined using two-way analysis of variance (ANOVA) with repeated measurements to compare values of the two groups (two levels) between the time configurations (four levels). In all cases, $\alpha$ was set to 0.05 and statistical significance was accepted for $P<0.05$, with the Bonferroni correction applied post hoc.

\section{Results}

\section{Experiments}

At the 0.05 significance level, the exponential model $\left(R^{2}=\right.$ 0.99714 ) was more likely to be correct than the linear model 
Table 1

List of experiments and details of testing protocols.

\begin{tabular}{|c|c|c|c|c|c|c|c|c|c|}
\hline \multirow[t]{2}{*}{ Experiment } & \multirow[t]{2}{*}{ Lubricant } & \multirow[t]{2}{*}{$n$} & \multirow[t]{2}{*}{ Test } & \multirow[t]{2}{*}{ Extension angle at the start $\left(^{\circ}\right)$} & \multirow[t]{2}{*}{ Load $(N)$} & \multicolumn{4}{|c|}{ Post mortem time of test (min) } \\
\hline & & & & & & $\begin{array}{l}\mathrm{t} 1 \\
90 \\
\end{array}$ & $\begin{array}{l}\mathrm{t} 2 \\
190\end{array}$ & $\begin{array}{l}\text { t3 } \\
290\end{array}$ & $\begin{array}{l}\mathrm{t} 4 \\
390 \\
\end{array}$ \\
\hline Experiment 1 & Synovial fluid & 6 & Pendulum test & 5 & 400 & + & + & + & + \\
\hline Experiment 2 & Synovial fluid & 6 & Pendulum test & 5 & 400 & + & & & \\
\hline Experiment 2 & Synovial fluid + alcohol & & Pendulum test & 5 & 400 & + & + & + & + \\
\hline
\end{tabular}

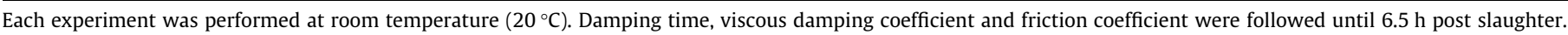

$\left(R^{2}=0.85849\right)$ to fit joint pendular decay (Fig. 3a and b). This confirmed that pendular joint damping is fluid and that the HOFD model provided the best representation of the whole joint. Measurements of $\tau, c$ and $\mu$ that were performed in experiments $\mathrm{E} 1$ and E2, are presented in Table 2 and in Fig. $4 \mathrm{a}-\mathrm{c}$.
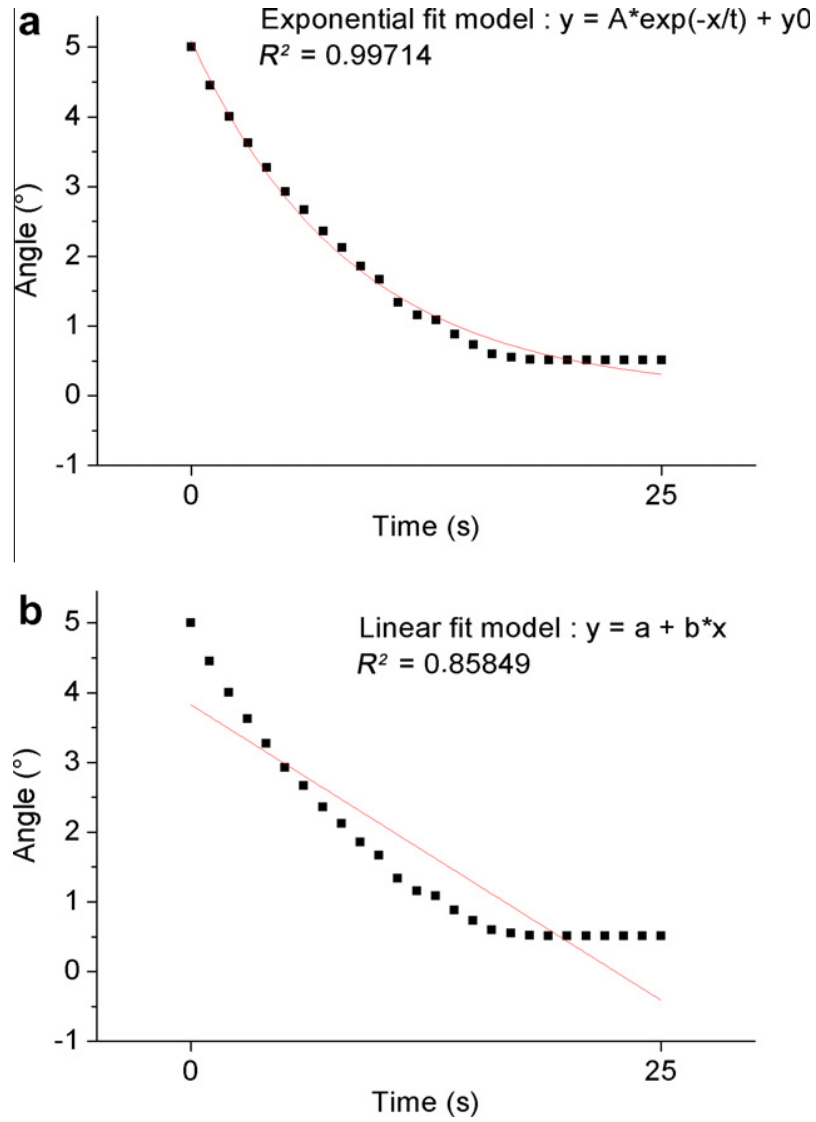

Fig. 3. Fitted joint pendular decay using an exponential model (a) and using a linear model (b).

\section{Effects of time and treatment}

In group 2, the comparison of $\tau, c$ and $\mu$ before and just after the alcohol injection showed that $\tau$ increased and that $c$ and $\mu$ decreased, but not significantly. In group 2, following alcohol injection, and in group 1 , the effect of time resulted in a significant ( $P<0.05)$ decrease in $\tau$, a significant $(P<0.05)$ increase in $c$ and a significant $(P<0.05)$ increase in $\mu$. Moreover, we found that the evolution of $\tau, c$ and $\mu$ were significantly $(P<0.05)$ different in group 1 compared to group 2. Indeed, $\tau$ decreased, $c$ and $\mu$ increased more rapidly and to a higher level in group 2 than in group 1 .

\section{Interaction of time and alcohol injection}

For $\tau$, the effect of alcohol injection within time was found to be significant $(P<0.05)$ except within 1 . Furthermore, the effect of time within group 1 was found to be significant $(P<0.05)$ in the $\mathrm{t} 1-\mathrm{t} 3, \mathrm{t} 1-\mathrm{t} 4$ and $\mathrm{t} 2-\mathrm{t} 4$ pair wise comparisons, whereas the effect of time within group 2 was found to be significant $(P<0.05)$ in the $\mathrm{t} 1-\mathrm{t} 2, \mathrm{t} 1-\mathrm{t} 3, \mathrm{t} 1-\mathrm{t} 4$ and $\mathrm{t} 2-\mathrm{t} 4$ pair wise comparisons. For $c$, the effect of time within group 2 was found to be significant $(P<0.05)$ in the $\mathrm{t} 1-\mathrm{t} 3$ and $\mathrm{t} 1-\mathrm{t} 4$ pair wise comparisons. For $\mu$, the effect of alcohol injection within time was found to be significant $(P<0.05)$ only in 44 and the effect of time within group 2 was found to be significant $(P<0.05)$ only in the $t 1-t 4$ pair wise comparison.

\section{Macroscopic examination}

In all the experiments, the articular surfaces were smooth, and stiff with a bluish tint. None showed an arthritic aspect that could influence the results on joint mechanical properties. The SF was found to be slightly clearer in group 2 than in group 1 .

\section{Discussion}

The main objective of our study was to measure joint viscous friction parameters $(\tau, c$ and $\mu$ ) using an equine pendulum test, and to investigate the influence of post mortem time and of the injection of alcohol on the evolution of $\tau, c$ and $\mu$.

Table 2

Mean values of friction coefficient $(\mu)$ viscous damping coefficient $(c)$ and damping time $(\tau)$, which were collected in experiments E1 and E2.

\begin{tabular}{|c|c|c|c|c|c|c|}
\hline \multirow{2}{*}{$\begin{array}{l}\text { Experiments } \\
\text { Parameters }\end{array}$} & \multicolumn{3}{|c|}{ Experiment 1} & \multicolumn{3}{|l|}{ Experiment 2} \\
\hline & $\mu\left(10^{-3}\right)$ & $c$ & $\tau$ & $\mu\left(10^{-3}\right)$ & c & $\tau$ \\
\hline $\mathrm{t} 1(90 \mathrm{~min})$ & $6.9(1.69)$ & $0.862(0.174)$ & $24.47(1.45)$ & $\begin{array}{l}5.69(1.26) \\
5.49(1.25)\end{array}$ & $\begin{array}{l}1.069(0.132) \\
0.945(0.133)\end{array}$ & $\begin{array}{l}22.56(2.00) \\
23.23(2.04)\end{array}$ \\
\hline $\mathrm{t} 2(190 \mathrm{~min})$ & 7 (1.57) & $0.914(0.186)$ & $23.99(1.33)$ & $7.23(1.68)$ & $1.6(0.257)$ & $19.72(1.74)$ \\
\hline t3 $(290 \mathrm{~min})$ & $7.3(1.64)$ & $0.972(0.132)$ & $22.44(1.23)$ & $7.76(1.84)$ & $1.951(0.400)$ & $17.97(1.58)$ \\
\hline t4 (390 min) & $7.9(1.69)$ & $1.095(0.151)$ & $21.46(1.00)$ & $14.10(5.58)$ & $2.313(0.509)$ & $17.49(1.56)$ \\
\hline
\end{tabular}

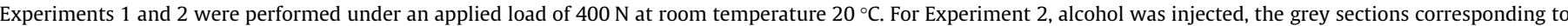

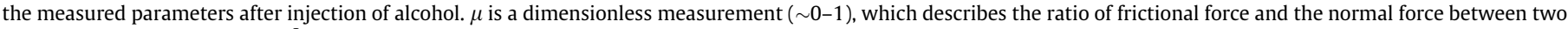
surfaces. The units for $c$ is $\mathrm{kg} \mathrm{m}^{2} / \mathrm{s}$ and for $\tau$ is second. The SE is shown in brackets. 


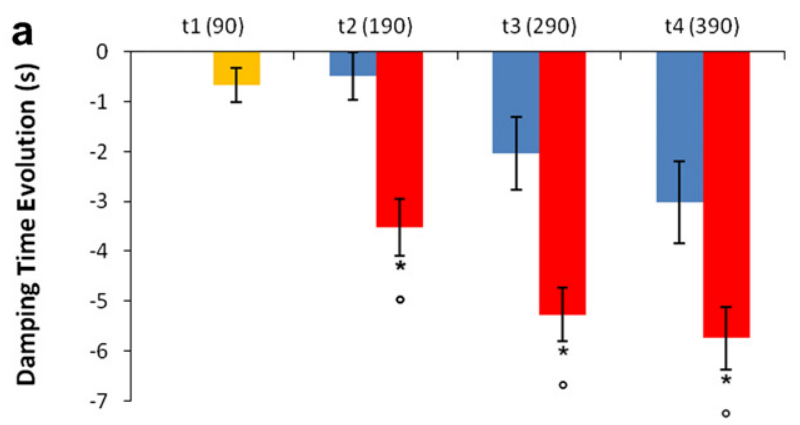

Post Mortem Time ( $\mathrm{min})$
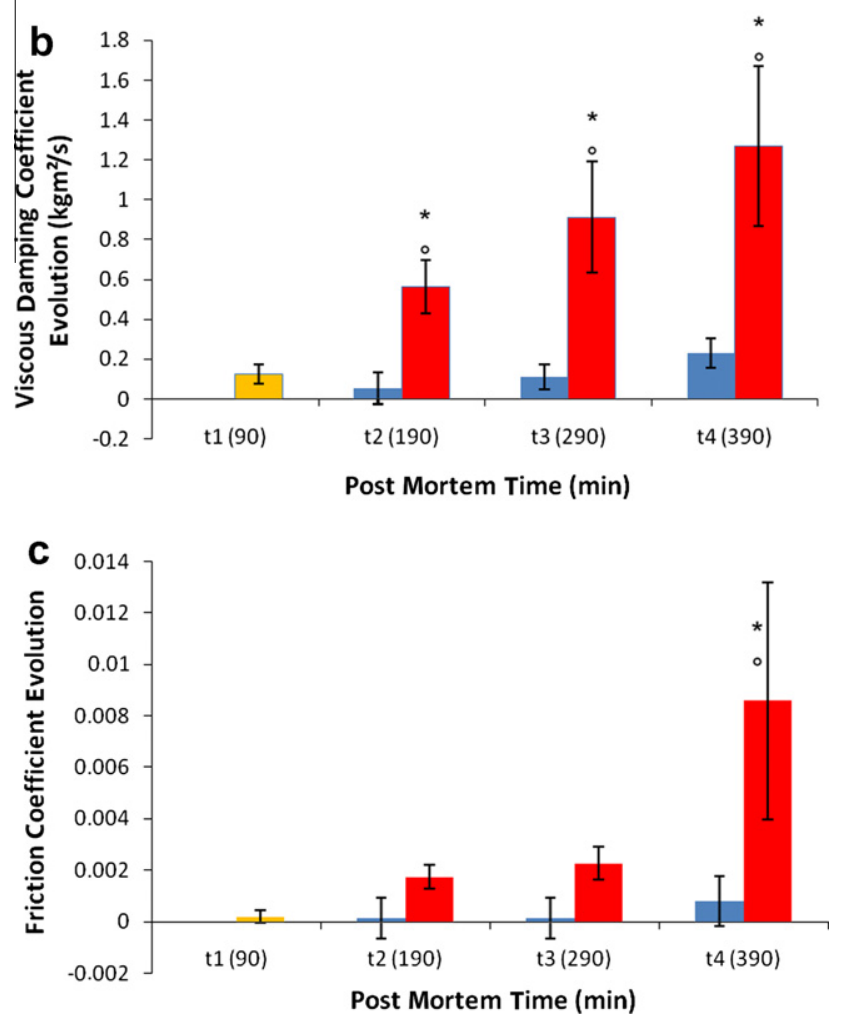

Fig. 4. Effect of alcohol on the damping time (a); on the viscous damping coefficient (b); on the friction coefficient (c) of the whole fetlock joint. The control group is shown in blue and the alcohol group is shown in yellow representing before alcohol injection and in red representing after alcohol injection. Each point represents the mean \pm SE; ${ }^{*} P<0.05$, significant difference within group; ${ }^{\circ} P<0.05$, significant difference between control and lipid solvent groups. The unit for $\tau$ is second, the units for $c$ is $\mathrm{kg} \mathrm{m}^{2} / \mathrm{s}$ and $\mu$ is dimensionless $(\sim 0-1)$.

It was not possible to compare the current results with other reports because this was the first time that a pendulum apparatus has been used as an equine MPJ model. Nevertheless, in comparing our results with joint pendular studies carried out in other species, our mean $\mu \quad(\sim 0.006)$ was similar to reports in other studies $(\sim 0.001-0.05)$, while our mean $c\left(\sim 0.96 \mathrm{~kg} \mathrm{~m}^{2} / \mathrm{s}\right)$ was not $(\sim 3 \times$ $10^{-5}-1.4 \times 10^{-3} \mathrm{~kg} \mathrm{~m}^{2} / \mathrm{s}$ ) (Crisco et al., 2007; Drewniak et al., 2009). This difference may be due to the substantially different load (ratio: 1/1000) used in the calculations.

The joint pendular decay was exponential, which agreed with other reports (Crisco et al., 2007; Drewniak et al., 2009), and demonstrated that the exponential decay function (including both frictional and viscous damping) dramatically increased the accuracy of measuring the frictional response in a pendulum test of modelled whole joints.

However, our experimental procedure had several limitations, including the fact that the joint pendulum test was performed at room temperature $\left(20^{\circ} \mathrm{C}\right)$ and specimens were not perfused (hypoxia). The conditions were not physiological and this may be responsible for a change in the rheological properties of the joint. Indeed, whereas our results clearly established that $\tau, c$ and $\mu$ changed during post mortem time in both groups, this could be explained by the decrease in temperature, the deteriorating hydration status and post mortem autolysis. The results highlight the importance of rigorous and consistent specimen preparation when evaluating joint mechanical properties, which should only be tested on fresh cadavers at the same post mortem time.

In group 2, the comparison of $\tau, c$ and $\mu$ before and just after alcohol injection showed that $\tau$ increased, and that $c$ and $\mu$ decreased, but not significantly. This result could be explained by the fact that the ratio of the volume of alcohol (constant) to the MPJ volume (variable) was randomised, and so the dilution effect was not significant. A comparison of the control group (group 1) and the alcohol group (group 2 ) showed a significant difference between the evolution of $\tau, c$ and $\mu$, where $\tau$ decreased and $c$ and $\mu$ increased more rapidly and to a higher level in the alcohol group than in the control group.

Assuming that the addition of alcohol had only a lipid solvent effect on the SF, the results of our experiments suggest that SAPLs contribute to boundary lubrication of the joint. However, Schmidt et al. (2007) demonstrated that SF friction coefficients were not significantly affected by the addition of the dipalmitoyl-phosphatidylcholine (DPPC) at physiological concentrations. So, while the cartilage samples in that experiment were completely immersed in the test lubricant for $24 \mathrm{~h}$ prior to lubrication testing, SAPL organisation was probably disturbed by the ambient air condition. Moreover, Chen et al. (2007) showed that the individual relative percentage of DPPC on all SAPLs was only $8 \%$, and its ability to reduce the frictional response was lower than other SAPLs (Chen et al., 2005). Finally, it should also be noted that the lipid boundary lubricant of synovial joints is constituted by a combination of SAPLs rather than a single SAPL (Sarma et al., 2001).

A further consideration is that the triplex (cartilage-SF-cartilage) is linked structurally and functionally and the frictional response of cartilage is not limited to a surface phenomenon and is greatly influenced by the degree of tissue degradation (Basalo et al., 2005). Indeed, experimental cartilage damage has suggested a molecular level of matrix degradation associated with a deterioration in the functional physical properties of the tissue, such as a decrease in the compressive stiffness (Bonassar et al., 1995), a decrease in Young's modulus (Lyyra et al., 1999), and alterations in the frictional response (Basalo et al., 2005).

Our previous work has suggested the same conclusion regarding cartilage ageing (Noble et al., 2009). Therefore, taking into consideration the fact that the injection of alcohol not only had a lipid solvent effect on the SF but also dehydration and an oxidant effects on the triplex, these experiments suggested that the lubrication mechanism of the alcohol group could have been compromised. A combination of SAPL disturbance, dehydration and oxidant effects could have limited the function of HA and lubricin, so destabilising SF rheology since antioxidant effects and boundary lubrication did not occur. The biphasic boundary lubrication theory would suggest that the cartilage lost its matrix integrity so that the interstitial pressure could have fallen and the viscous frictional response could have increased, although further studies are required to confirm this.

\section{Conclusions}

This was the first time that viscous friction parameters have been measured using an equine pendulum. Experimental data con- 
firmed that pendular joint damping can be modelled by a harmonic oscillator with fluid damping. In addition, lipid solvent treatment was found to be responsible for upper viscous friction parameters and for a reduced damping time, which suggests that the articular lubricating ability was compromised. Although the pendulum is a simplified tribological model, it may be useful for testing the efficacy of various bio-lubricant treatments.

\section{Conflict of interest statement}

None of the authors of this paper has a financial or personal relationship with other people or organisations that could inappropriately influence or bias the content of the paper.

\section{References}

Ateshian, G.A., 1997. A theoretical formulation for boundary friction in articular cartilage. Journal of Biomechanical Engineering 119, 81-86.

Ateshian, G.A., 1998. The role of interstitial fluid pressurization and surface porosities on the boundary friction of articular cartilage. Journal of Tribology $120,241-251$.

Ateshian, G.A., 2009. The role of interstitial fluid pressurization in articular cartilage lubrication. Journal of Biomechanics 42, 1163-1176.

Basalo, I.M., Raj, D., Krishnan, R., Chen, F.H., Hung, C.T., Ateshian, G.A., 2005. Effects of enzymatic degradation on the frictional response of articular cartilage in stress relaxation. Journal of Biomechanics 38, 1343-1349.

Bell, C.J., Ingham, E., Fisher, J., 2006. Influence of hyaluronic acid on the timedependent friction response of articular cartilage under different conditions. Proceedings of the Institution of Mechanical Engineers [H] 220, 23-31.

Bonassar, L.J., Frank, E.H., Murray, J.C., Paguio, C.G., Moore, V.L., Lark, M.W., Sandy, J.D., Wu, J.J., Eyre, D.R., Grodzinsky, A.J., 1995. Changes in cartilage composition and physical properties due to stromelysin degradation. Arthritis and Rheumatism 38, 173-183.

Caligaris, M., Ateshian, G.A., 2008. Effects of sustained interstitial fluid pressurization under migrating contact area, and boundary lubrication by synovial fluid, on cartilage friction. Osteoarthritis and Cartilage 16, 1120-1127.

Chen, Y., Crawford, R.W., Oloyede, A., 2007. Unsaturated phosphatidylcholines lining on the surface of cartilage and its possible physiological roles. Journal of Orthopaedic Surgery 2, 14.

Chen, Y., Hills, B.A., Hills, Y.C., 2005. Unsaturated phosphatidylcholine and its application in surgical adhesion. ANZ Journal of Surgery 75, 1111-1114.

Crisco, J.J., Blume, J., Teeple, E., Fleming, B.C., Jay, G.D., 2007. Assuming exponential decay by incorporating viscous damping improves the prediction of the coefficient of friction in pendulum tests of whole articular joints. Proceedings of the Institution of Mechanical Engineers [H] 221, 325-333.

Drewniak, E.I., Jay, G.D., Fleming, B.C., Crisco, J.J., 2009. Comparison of two methods for calculating the frictional properties of articular cartilage using a simple pendulum and intact mouse knee joints. Journal of Biomechanics 42, 19961999.

Elsaid, K.A., Jay, G.D., Warman, M.L., Rhee, D.K., Chichester, C.O., 2005. Association of articular cartilage degradation and loss of boundary-lubricating ability of synovial fluid following injury and inflammatory arthritis. Arthritis and Rheumatism 52, 1746-1755.

Forsey, R.W., Fisher, J., Thompson, J., Stone, M.H., Bell, C., Ingham, E., 2006. The effect of hyaluronic acid and phospholipid based lubricants on friction within a human cartilage damage model. Biomaterials 27, 4581-4590.
Foy, J.R., Williams, P.F., Powell, G.L., Ishihara, K., Nakabayashi, N., Laberge, M., 1999. Effect of phospholipidic boundary lubrication in rigid and compliant hemiarthroplasty models. Proceedings of the Institution of Mechanical Engineers [H] 213, 5-18.

Grimshaw, M.J., Mason, R.M., 2000. Bovine articular chondrocyte function in vitro depends upon oxygen tension. Osteoarthritis and Cartilage 8, 386-392.

Higaki, H., Murakami, T., Nakanishi, Y., 1997. Lubricating ability of LangmuirBlodgett films as boundary lubricating films on articular surfaces. Japan Society of Mechanical Engineers International Journal 40, 776-781.

Jay, G.D., 1992. Characterization of a bovine synovial fluid lubricating factor. I. Chemical, surface activity and lubricating properties. Connective Tissue Research 28, 71-88.

Krishnan, R., Kopacz, M., Ateshian, G.A., 2004. Experimental verification of the role of interstitial fluid pressurization in cartilage lubrication. Journal of Orthopaedic Research 22, 565-570.

Lin, D.C., Rymer, W.Z., 1991. A quantitative analysis of pendular motion of the lower leg in spastic human subjects. IEEE Transactions on Biomedical Engineering 38, 906-918.

Lyyra, T., Arokoski, J.P., Oksala, N., Vihko, A., Hyttinen, M., Jurvelin, J.S., Kiviranta, I., 1999. Experimental validation of arthroscopic cartilage stiffness measurement using enzymatically degraded cartilage samples. Physics in Medicine and Biology 44, 525-535.

Noble, P., Collin, B., Lecomte-Beckers, J., Magnee, A., Denoix, J.M., Serteyn, D., 2009 An equine joint friction test model using a cartilage-on-cartilage arrangement. The Veterinary Journal 2, 148-152.

Rhee, D.K., Marcelino, J., Baker, M., Gong, Y., Smits, P., Lefebvre, V., Jay, G.D., Stewart, M., Wang, H., Warman, M.L., Carpten, J.D., 2005. The secreted glycoprotein lubricin protects cartilage surfaces and inhibits synovial cell overgrowth. Journal of Clinical Investigation 115, 622-631.

Sarma, A.V., Powell, G.L., LaBerge, M., 2001. Phospholipid composition of articular cartilage boundary lubricant. Journal of Orthopaedic Research 19, 671-676.

Schmidt, T.A., Gastelum, N.S., Nguyen, Q.T., Schumacher, B.L., Sah, R.L., 2007. Boundary lubrication of articular cartilage: role of synovial fluid constituents. Arthritis and Rheumatism 56, 882-891.

Schneider, N., Mouithys-Mickalad, A., Lejeune, J.P., Duyckaerts, C., Sluse, F., DebyDupont, G., Serteyn, D., 2007. Oxygen consumption of equine articular chondrocytes: influence of applied oxygen tension and glucose concentration during culture. Cell Biology International 31, 878-886.

Schvartz, I., Seger, D., Shaltiel, S., 1999. Vitronectin. International Journal of Biochemistry and Cell Biology 31, 539-544.

Schwarz, I.M., Hills, B.A., 1998. Surface-active phospholipid as the lubricating component of lubricin. British Journal of Rheumatology 37, 21-26.

Tadmor, R., Chen, N., Israelachvili, J.N., 2002. Thin film rheology and lubricity of hyaluronic acid solutions at a normal physiological concentration. Journal of Biomedical Materials Research 61, 514-523.

Teeple, E., Elsaid, K.A., Fleming, B.C., Jay, G.D., Aslani, K., Crisco, J.J., Mechrefe, A.P., 2008. Coefficients of friction, lubricin, and cartilage damage in the anterior cruciate ligament-deficient guinea pig knee. Journal of Orthopaedic Research 26, 231-237.

Teeple, E., Fleming, B.C., Mechrefe, A.P., Crisco, J.J., Brady, M.F., Jay, G.D., 2007. Frictional properties of Hartley guinea pig knees with and without proteolytic disruption of the articular surfaces. Osteoarthritis and Cartilage 15, 309-315.

Tolosano, E., Altruda, F., 2002. Hemopexin: structure, function, and regulation. DNA and Cell Biology 21, 297-306.

Trunfio Sfarghiu, A., 2006. Modèle bio-tribologique des articulations. Rôle mécanique et physicochimique des assemblages moléculaires du fluide synovial (Phd thesis). In: Biomechanic Phd. Institut National des Sciences Appliquées, Lyon, p. 154. 